\title{
In Vivo Antiplasmodial Activity of Fractions and Chromatographic Sub Fractionions of Ethanol Extract of Clerodendrum Myricoides Leaves
}

\author{
Gebremichael Gebretsadik $^{1}$, Yalemtsehay Mekonnen ${ }^{2}$ \\ ${ }^{1}$ Department of Biology, College of Natural and Computational Sciences, Assosa University, Assosa, Ethiopia \\ ${ }^{2}$ Department of Biology, College of Natural Sciences, Addis Ababa University, Addis Ababa, Ethiopia
}

Email address:

mikigebre73@gmail.com (G. Gebretsadik),yalem_mekonnen_00@yahoo.com (Y. Mekonnen)

\section{To cite this article:}

Gebremichael Gebretsadik, Yalemtsehay Mekonnen. In Vivo Antiplasmodial Activity of Fractions and Chromatographic Sub Fractionions of Ethanol Extract of Clerodendrum Myricoides Leaves. Journal of Drug Design and Medicinal Chemistry. Vol. 2, No. 6, 2016 , pp. 60-64. doi: 10.11648/j.jddmc.20160206.11

Received: June 2, 2016; Accepted: July 15, 2016; Published: November 3, 2016

\begin{abstract}
Herbal medicine preparations from medicinal plants to treat malaria are potential source of new anti-malarial drug development. The objective of this study was to evaluate antiplasmodial activity of fractions and sub fractions of the ethanol extract of Clerodendrum myricoides leaves using 4-day suppressive test against Plasmodium berghei in Swiss albino mice. Methanol fraction (MF) and ethyl acetate fraction (EF) obtained from the successive fractionation of ethanol crude extract of C. myricoides showed highest activity with suppression of $77.24 \%$ and $65.21 \%$ at an oral dose of $300 \mathrm{mg} / \mathrm{kg} / \mathrm{day}$ respectively. Further, bioactivity guided fractionation (BGF) of ethanol extract provided some fractions which exhibited good antiplasmodial activity. From these, Hexane sub fraction (Hsf) was the most active that induced suppression of parasitaemia by $40.34 \%$ and $46.56 \%$ at $50 \mathrm{mg} / \mathrm{kg}$ and $100 \mathrm{mg} / \mathrm{kg}$ doses respectively. Hsf chromatographic sub fractions also induced suppression of parasitaemia by 45.89 and $50.53 \%$ at $20 \mathrm{mg} / \mathrm{kg}$ and $40 \mathrm{mg} / \mathrm{kg}$ of Hsf-5, respectively, and by 38.61 and $44.12 \%$ at $20 \mathrm{mg} / \mathrm{kg}$ and $40 \mathrm{mg} / \mathrm{kg}$ of Hsf-14, respectively. Bioactivity guided fractionation resulted in loss of antiplasmodial activity of the fractions and sub fractions and this leads to the conclusion that the activity of the plant leaves was due to synergistic effect of many active compounds.
\end{abstract}

Keywords: Antiplasmodial Activity, C. Myricoides, Plasmodium Berghei, Medicinal Plants, Traditional Medicine

\section{Introduction}

An estimated 3.4 billion people are at risk of malaria, of whom 1.2 billion are at high risk. $90 \%$ of all malaria deaths occur in sub-Saharan Africa [1]. Chemotherapeutic agents will continue to be in demand for the complete management of malaria [2], and the issue of resistance means that discovering new antimalarial drugs is an urgent priority [3]. Research on new antimalarials from plant products is the principal approach for discovery of new drugs with novel modes of action [4]. This approach is believed to exploit plant products that represent a virtually inexhaustible reservoir of molecules with enormous structural and chemical diversity [5]. The relevance of this approach is evidenced from the effective antimalarial activity of the two plant-based drugs, quinine and artemisinin [6]. This has generated much interest to explore the curing capacity of plants possessed by inhabitants of malaria endemic regions for their possible antimalarial efficacy. Hence, in the present study leaves of $C$. myricoides that is traditionally used to treat malaria was evaluated in Plasmodium berghei infected Swiss albino mice.

\section{Materials and Methods}

\subsection{Plant Material}

C. myricoides (Lamiaceae) was collected from Eastern part of Addis Ababa, Ethiopia. Identification and authentication of the plant specimen was done at the National Herbarium of Addis Ababa University by a botanist and voucher specimen 
(GG4/2011) was deposited in the Herbarium. The collected leaves of $C$. myricoides were air dried under shade for about two weeks.

\subsection{Plant Extraction and BGF of Ethanol Crude Extract}

\subsubsection{Preparation Crude Extract and Fractionation}

The crude extract was prepared by maceration of the powder in ethanol $(99.5 \%)$ at room temperature [7]; by placing $1 \mathrm{~kg}$ of powdered plant material in an Erlenmeyer flask containing $6 \mathrm{~L}$ of absolute ethanol and it was placed on orbital shaker (at $120 \mathrm{rpm}$ ) for 24 hours at room temperature. Then filtered out using a Whatman filter paper that yielded a greenish filtrate, which was concentrated under reduced pressure in a rotary evaporator (Buchi type TRE 121, Switzerland) to give $185 \mathrm{~g}(18.5 \%)$ dark semi-solid ethanol crude extract.

The ethanol extract was subjected to successive fractionation using n-hexane to obtain hexane fraction (HF) followed by ethyl acetate to obtain ethyl acetate fraction (EF). The marc that remained was methanol soluble (MF). All the fractions were stored at $-20^{\circ} \mathrm{C}$ until they were subjected to anti-malarial test. Following in vivo antimalarial evaluation the fractions that were found to be active (EF and $\mathrm{MF}$ ) were further fractionated using solvent partitioning and column chromatography (CC) respectively. The extraction and fractionation were conducted in the laboratory of the Natural Products Chemistry, Chemistry Department, Addis Ababa University.

Further sub fractionation was done by dissolving $5 \mathrm{~g}$ of the $\mathrm{EF}$ fraction in $\mathrm{MeOH}(50 \mathrm{ml})$ and its volume was reduced to about $30 \mathrm{ml}$. Then $30 \mathrm{ml}$ dist. water was added to make $50 \%$ aqueous solution. It was fractionated three times $(3 \mathrm{x})$ each with $20 \mathrm{ml}$ of $\mathrm{n}$-hexane to obtain hexane sub fraction (Hsf). The aqueous phase that remained after fractionated with $n$-hexane was further fractionated $3 x$ each with $20 \mathrm{ml}$ of chloroform to obtain chloroform sub fraction (Csf) and aqueous phase. Finally the aqueous phase that remained after extracted with chloroform was partitioned using n-butanol to obtain n-butanol sub fraction (Bsf). Each sub fraction was concentrated in rotary evaporator to yield $3.6 \mathrm{~g}(72 \%), 1 \mathrm{~g}(20 \%)$ and $0.3 \mathrm{~g}(6 \%)$ of Hsf, Csf and Bsf respectively.

\subsubsection{Column Chromatographic Fractionation of MF and Hsf}

The marc, MF (5 g), was dissolved in methanol and subjected to column chromatography. Elution was done using cyclohexane: dichloromethane (DCM): $\mathrm{MeOH}$ solvents with different ratios (100:0:0, 1:1:0, 0:19:1, 0:1:1, $0: 1: 3,0: 1: 9)$ by increasing polarity. Similarly Hsf (3.6 g) was adsorbed onto silica gel and applied on top of column chromatography packed with fifty $(50 \mathrm{~g})$ grams of silica gel (230-400 mesh). Elusion was done using hexane: EtOAc and EtOAc: $\mathrm{MeOH}$ solvents with different ratios (1:0, 9:1, $4: 1,7: 3,1: 1,3: 7,1: 4,0: 1,1: 0,4: 1)$ by increasing polarity. At the end the eluted fractions were combined based on their TLC profiles.

\subsection{In vivo Antiplasmodial Activity of Fractions and Subfractions of C. Myricoides}

\subsubsection{Experimental Animals}

Male Swiss albino mice weighing 25-35 grams, 6-8 weeks of age obtained from the Animal House of the College of Natural Sciences, Addis Ababa University, were used in the study. The animals were housed in standard cages and acclimatized for a period of 10 days before using for the experiment.

\subsubsection{Malaria Parasite and Inoculation}

For the in vivo antiplasmodial assay of extracts (plant fractions, sub fractions and standard drug) the mouse infective CQ sensitive strain of $P$. berghei maintained in Biomedical laboratory of the College of Natural Sciences was used. The parasites were maintained by serial passage of blood obtained from the tail end of infected mice to noninfected ones on weekly basis. Each mouse used in the experiment was infected intraperitoneally with $0.2 \mathrm{ml}$ of infected blood containing about $1 \times 10^{6}-10^{7} P$. bergheiparasitized erythrocytes [8]. For each experiment about $1 \mathrm{ml}$ $P$. berghei infected blood sample was obtained by gentle cardiac puncture of the donor mouse with rising parasitaemia of about $25-35 \%$ in such a way that $1 \mathrm{ml}$ blood contains $5 \times 10^{6}-10^{7} P$. berghei-parasitized erythrocytes per $\mathrm{ml}[6,9]$.

\subsubsection{Evaluation of Suppressive Activity of the Fractions}

The evaluation protocol was based on Peters'4-day suppressive test against $P$. berghei infection in mice [10]. Twenty infected mice were randomly divided into two test groups and two control groups (each for chloroquine (CQ) as a standard drug and dimethyl sulfoxide (DMSO) as a negative control) for each of the fractions as well as sub fractions: HF, EF, MF, EtOAc sub fractions (Hsf, Csf and Bsf) and the chromatographic sub fractions of MF and Hsf (MF-3, MF-4, MF-5, MF-6; Hsf-1, Hsf-5, Hsf-7, Hsf-8 and Hsf-14) respectively.

Five mice for each cage of the test groups and the control groups were assigned. The test fractions were prepared in two doses $(150 \mathrm{mg} / \mathrm{kg}$ and $300 \mathrm{mg} / \mathrm{kg}$ of body weight for HF, $\mathrm{EF}$ and MF; $50 \mathrm{mg} / \mathrm{kg}$ and $100 \mathrm{mg} / \mathrm{kg}$ of body weight for Hsf, Csf and Bsf; $40 \mathrm{mg} / \mathrm{kg}$ and $20 \mathrm{mg} / \mathrm{kg}$ of body weight for Hsf-1, Hsf-5, Hsf-7, Hsf-8 and Hsf-14,); CQ at $25 \mathrm{mg} / \mathrm{kg}$ in a volume of $0.2 \mathrm{ml}$ and the negative control group was given the solvent $(0.2 \mathrm{ml}$ of $20 \% \mathrm{DMSO})$ for the same duration. All the test plant fractions, CQ and DMSO used in the antiplasmodial study were administered as a single dose per day through intragastric route by using standard intragastric gavage to insure safe ingestion. Within 3 hours postinoculation of mice with the parasites, on day 0 (D0) treatments of infected mice were started and continued daily for four days (i.e. from D0 to day 3) in 24 hour schedule.

On the fifth day (D4) blood samples were collected from tail snip of each mouse and smeared on to microscopic slides to make thin blood smear. The thin blood smear first fixed with methanol for 30 seconds and stained with $10 \%$ Geimsa solution, $\mathrm{pH} \mathrm{7.2,} \mathrm{for} 25 \mathrm{~min}$. Then, five uniform fields from 
tailed region of each stained slide (for each mouse) were examined under the microscope with an oil immersion objective of $100 \times$ magnification power to evaluate the percent suppression of each fraction with respect to the control groups [9].

Then percent parasitaemia and suppression were calculated for each dose by comparing the parasitaemia in treated group with negative control group according to the following formula [11].

Percentage parasitemia (Pra.) in each field was calculated as:

Total number of $\mathrm{PRBC} \times 100$

Total number of RBC

Where,

$\mathrm{PRBC}=$ Parasitized Red Blood Cells

$\mathrm{RBC}=$ Red Blood Cells

Percentage suppression was calculated as:

Par. in control (\%)-Par. in treated group $(\%) \times 100$

Parasitemia in control (\%)

\section{Results}

The fractions and sub fractions did not eradicate parasites completely, but the percentage of parasitaemia was lower in all the mice administered with the fractions and sub fractions of the $C$. myricoides leaves ethanol extract as compared to the negative control groups that were given the vehicle only. The test fractions and sub fractions which showed greater than $30 \%$ suppression were considered to be active [12].
Statistical analysis using Scheffe's procedure indicated that groups of mice treated with $150 \mathrm{mg} / \mathrm{kg}$ and $300 \mathrm{mg} / \mathrm{kg} \mathrm{MF}$ and EF of the leaves of $C$. myricoides ethanol extract showed statistically significant difference in parasitaemia level as compared to the negative control group $(\mathrm{P}<0.05)$. MF at doses of $150 \mathrm{mg} / \mathrm{kg}$ and $300 \mathrm{mg} / \mathrm{kg}$ showed a suppressive effect of $72.90 \%$ and $77.24 \%$ respectively, whereas EF at doses of $150 \mathrm{mg} / \mathrm{kg}$ and $300 \mathrm{mg} / \mathrm{kg}$ showed a suppressive effect of $59.88 \%$ and $65.21 \%$, respectively, which have statistically significant difference as compared to the negative control $(\mathrm{P}<0.05)$. It was observed that there was a slight increase of percentage suppression with increase in fraction concentration.

On the other hand, the mice administered with HF of $C$. myricoides leaves ethanol extract did not show significant parasitaemia suppression as compared to the mice in the control group ( $\mathrm{P}>0.05)$ (Table 1). The MF chromatographic subfractions also induced suppression of parasitaemia by 40.95 and $48.14 \%$ at $50 \mathrm{mg} / \mathrm{kg}$ and $100 \mathrm{mg} / \mathrm{kg}$ of $\mathrm{MF}-5$, respectively, and by 45.07 and $51.03 \%$ at $50 \mathrm{mg} / \mathrm{kg}$ and 100 $\mathrm{mg} / \mathrm{kg}$ of MF-6, respectively (Table 2).

Hsf induced suppression of parasitaemia by $40.34 \%$ and $46.56 \%$ at $50 \mathrm{mg} / \mathrm{kg}$ and $100 \mathrm{mg} / \mathrm{kg}$ doses respectively. The chloroform and $n$ buthanol subfractions of EF did not show significant reduction in parasitaemia $(\mathrm{P}>0.05)$ (Table 3$)$. Hsf chromatographic sub fractions also induced suppression of parasitaemia by 45.89 and $50.53 \%$ at $20 \mathrm{mg} / \mathrm{kg}$ and $40 \mathrm{mg} / \mathrm{kg}$ of Hsf-5, respectively, and by 38.61 and $44.12 \%$ at $20 \mathrm{mg} / \mathrm{kg}$ and 40 $\mathrm{mg} / \mathrm{kg}$ of Hsf-14, respectively (Table 4).

Table 1. The antiplasmodial effect of $M F, E F$ and $H F$ of $C$. myricodes ethanol extract against $P$. berghei in mice.

\begin{tabular}{|c|c|c|c|}
\hline \multirow{2}{*}{ Fractions of C. myricoides ethanol extract } & \multirow{2}{*}{ Dose (mg/kg/day) } & \multicolumn{2}{|l|}{ Antiplasmodial activity } \\
\hline & & $\%$ Parasitaemia + SEM & \% Suppression \\
\hline \multirow{3}{*}{ Methanol fraction (MF) } & $\mathrm{NC}$ & $35.47 \pm 0.46^{\mathrm{a}}$ & 0.00 \\
\hline & 150 & $9.61 \pm 0.38^{\mathrm{b}}$ & 72.90 \\
\hline & 300 & $8.07 \pm 0.39^{\mathrm{b}}$ & 77.24 \\
\hline \multirow{3}{*}{ Ethyl acetate (EF) } & $\mathrm{NC}$ & $35.47 \pm 0.46^{\mathrm{a}}$ & 0.00 \\
\hline & 150 & $14.23 \pm 0.34^{\mathrm{b}}$ & 59.88 \\
\hline & 300 & $12.34 \pm 0.45^{\mathrm{b}}$ & 65.21 \\
\hline \multirow{3}{*}{ Hexane (HF) } & $\mathrm{NC}$ & $28.48 \pm 0.41^{\mathrm{a}}$ & 0.00 \\
\hline & 150 & $24.60 \pm 0.84^{\mathrm{a}}$ & 15.77 \\
\hline & 300 & $23.51 \pm 0.64^{\mathrm{a}}$ & 17.45 \\
\hline
\end{tabular}

$\mathrm{a}, \mathrm{b}=$ Values in the same column followed by the same letter do not differ significantly $(\mathrm{P}>0.05)$.

Table 2. The antiplasmodial effect of chromatographic subfractions of MF of $C$. myricoides leaves against P. berghei in mice.

\begin{tabular}{llll}
\hline \multirow{2}{*}{ Chromatographic subfractions of MF } & \multirow{2}{*}{ Dose (mg/kg/day) } & \multicolumn{2}{c}{ Antiplasmodial activity } \\
\cline { 3 - 4 } & & \% Parasitaemia + SEM & \% Suppression \\
\hline \multirow{2}{*}{ MF-3 } & NC & $31.55 \pm 1.60^{\mathrm{a}}$ & 0.00 \\
& 50 & $30.54 \pm 0.41^{\mathrm{a}}$ & 3.29 \\
MF-4 & 100 & $30.27 \pm 0.52 \mathrm{a}$ & 4.05 \\
& NC & $31.55 \pm 1.603 \mathrm{a}$ & 0.00 \\
MF-5 & 50 & $30.17 \pm 0.32 \mathrm{a}$ & 9.37 \\
& 100 & $28.51 \pm 0.43 \mathrm{a}$ & 0.00 \\
MF-6 & NC & $31.55 \pm 1.603 \mathrm{a}$ & 40.95 \\
& 100 & $18.63 \pm 0.37^{\mathrm{b}}$ & 48.14 \\
\end{tabular}

$\mathrm{a}, \mathrm{b}=$ Values in the same column followed by the same letter do not differ significantly $(\mathrm{P}>0.05)$. 
Table 3. The antiplasmodial activity of Hsf, Csf, and Bsf of EF of C. myricoides leaves against P. berghei in mice.

\begin{tabular}{llll}
\hline \multirow{2}{*}{ Subfractions of EF } & Dose (mg/kg/day) & Antiplasmodial activity & \% Suppression \\
\cline { 3 - 4 } & & \% Parasitaemia+SEM & 0.00 \\
Hexane subfraction (Hsf) & NC & $27.47+0.48^{\mathrm{a}}$ & 40.34 \\
& 50 & $16.37+0.49^{\mathrm{b}}$ & 46.56 \\
& 100 & $14.11+0.51^{\mathrm{b}}$ & 0.00 \\
Chloroform subfraction (Csf) & $\mathrm{NC}$ & $25.40+0.71^{\mathrm{a}}$ & 7.08 \\
& 50 & $23.60+1.02^{\mathrm{a}}$ & 7.91 \\
Buthanolsubfraction (Bsf) & 100 & $23.39+1.28^{\mathrm{a}}$ & 0.00 \\
& $\mathrm{NC}$ & $27.47+0.48^{\mathrm{a}}$ & 26.02 \\
\hline
\end{tabular}

$\mathrm{a}, \mathrm{b}=$ Values in the same column followed by the same letter do not differ significantly $(\mathrm{P}>0.05)$.

Table 4. Antiplasmodial activity of chromatographic subfractions of Hsf of C. myricoides leaves against P. berghei in mice.

\begin{tabular}{|c|c|c|c|}
\hline \multirow{2}{*}{ Chromatographic subfractions of Hsf } & \multirow{2}{*}{ Dose (mg/kg/day) } & \multicolumn{2}{|l|}{ Antiplasmodial activity } \\
\hline & & $\%$ Parasitaemia + SEM & \% Suppression \\
\hline \multirow{3}{*}{ Hsf-1 } & $\mathrm{NC}$ & $33.84 \pm 0.33^{\mathrm{a}}$ & 0.00 \\
\hline & 20 & $28.87 \pm 0.36^{\mathrm{a}}$ & 14.68 \\
\hline & 40 & $27.23 \pm 0.29^{\mathrm{a}}$ & 19.53 \\
\hline \multirow{3}{*}{ Hsf-5 } & $\mathrm{NC}$ & $33.84 \pm 0.33^{\mathrm{a}}$ & 0.00 \\
\hline & 20 & $18.31 \pm 0.39^{\mathrm{b}}$ & 45.89 \\
\hline & 40 & $16.74 \pm 0.36^{\mathrm{b}}$ & 50.53 \\
\hline \multirow{3}{*}{ Hsf-7 } & $\mathrm{NC}$ & $33.84 \pm 0.33^{\mathrm{a}}$ & 0.00 \\
\hline & 20 & $29.80 \pm 0.47^{\mathrm{a}}$ & 11.93 \\
\hline & 40 & $27.76 \pm 0.34^{\mathrm{a}}$ & 17.96 \\
\hline \multirow{3}{*}{ Hsf-8 } & $\mathrm{NC}$ & $30.14 \pm 0.65^{\mathrm{a}}$ & 0.00 \\
\hline & 20 & $27.56 \pm 0.58^{\mathrm{a}}$ & 8.56 \\
\hline & 40 & $25.96 \pm 0.61^{\mathrm{a}}$ & 13.86 \\
\hline \multirow{3}{*}{ Hsf-14 } & $\mathrm{NC}$ & $30.14 \pm 0.65^{\mathrm{a}}$ & 0.00 \\
\hline & 20 & $18.50 \pm 0.33^{b}$ & 38.61 \\
\hline & 40 & $16.84 \pm 0.67^{b}$ & 44.12 \\
\hline
\end{tabular}

$\mathrm{a}, \mathrm{b}=$ Values in the same column followed by the same letter do not differ significantly $(\mathrm{P}>0.05)$.

\section{Discussion}

In the present study the ethanol crude extract of the leaves of $C$. myricodies showed antiplasmodial avtivity in $P$. berghei infected albino mice. The result of this study showed a suppressive effect of $77.24 \%$ and $65.21 \%$ at $300 \mathrm{mg} / \mathrm{kg}$ oral dose of methanol fraction and ethyl acetate fraction respectively. Moreover, at doses of $150 \mathrm{mg} / \mathrm{kg} \mathrm{MF}$ and EF showed a suppressive effect of $72.90 \%$ and $59.88 \%$ respectively. This result is in agreement with the study that reported suppressive effect of $69.31 \%$ and 61.30 at 150 $\mathrm{mg} / \mathrm{kg}$ oral dose of methanol fraction and ethyl acetate fraction respectively [13]. Furthermore, the methanol crude extract was found to show high activity, with suppressive effect of $82.25 \%$ at $600 \mathrm{mg} / \mathrm{kg}$ against $P$. bergei in mice as reported by reference [14] and with IC $50=16.8+2.65 \mu \mathrm{g} / \mathrm{ml}$ as demonstrated in reference [15].

These observations suggest that the active constituents in the fractions might be cytotoxic for $P$. berghei, thereby inhibiting their development to the erythrocytic stage. Moreover, these studies have confirmed $C$. myricoides is among the plants that displayed interesting antimalarial activity in vitro as well as in vivo systems. On the contrary, the HF reduced only in a limited percent the parasitaemia and was considered to be inactive [12]. This result is similar with the work of reference [13], which reported low suppressive effect of $19.84 \%$ at $150 \mathrm{mg} / \mathrm{kg}$ oral dose of hexane fraction, except slight difference. There was also a report by reference [15], which indicated $C$. myricoides hexane extract was found to exhibit limited antiplasmodial activity in vitro against $P$. falciparum with IC50 value greater than 100 $\mu \mathrm{g} / \mathrm{ml}$. This observation might indicate the antimalarial active components of the crude extract and fractions obtained from it might not have non polar [14] or medium polar properties.

Hexane subfraction obtained from EF induced an inhibition of parasitaemia significantly as compared to the negative control and hence considered to be active [12]. The parasitemia suppression of Hsf is reduced as compared to EF. This result might indicate that either the active components responsible for the suppression of parasitenia in EF treated mice are partitioned into Hsf, Csf and Bsf or the in active components of EF are important for the enhancement of antiplasmodial activity of EF.

In the present study, the MF and the sub fractions obtained from it (MF-5 and MF-6) and EF and the sub fractions obtained from it (Hsf, Hsf-5 and Hsf-14), which might be enriched in alkaloids, terpenoides, steroids, phenolics and flavonoides are found to show good suppression by acting synergistically against $P$. berghei in infected mice. Furthermore, the TLC results of these fractions showed the presence of many compounds which might inhibit $P$. berghei growth using different mode of action. Hsf-5 and Hsf-14 
obtained from the hexane sub fraction showed significant reduction of parasitaemia as compared to the negative control. The TLC results of Hsf-5 showed the presence of two spots which may correspond with two different compounds. Hence, the activity of the fraction is due to synergistic effect of two compounds, as the above fractions. The NMR spectrometer analysis of Hsf-5 leads to the isolation of one pure compound, which might be the major component of the sub fraction. It also could be the active compound responsible for antimalarial activity of EF in general or Hsf-5 in particular.

\section{Conclusions}

Though bioactivity guided fractionation of the ethanol extract yielded active fractions and sub fractions, which can exhibit good antiplasmodial activity against $P$. berghei, fractionation resulted in the reduction of antiplasmodial activity of the plant. This leads to the conclusion that the plant leaves antiplasmodial activity is due to synergistic effect and using in its crude form can provide result. Considering this a large percentage of Ethiopian plants have not been investigated chemically or pharmacologically, they remain a potential source of leads for possible drug development.

\section{Acknowledgements}

The authors acknowledge Prof. Ermias Dagne and Mr. Yadesa for the work done on column chromatography and other related techniques in the Chemistry laboratory of the Addis Ababa University and also express gratitude for the financial support of the School of Graduate Studies of the Addis Ababa University, Ethiopia.

\section{References}

[1] WHO, "World malaria report 2013" World Health Organization, Geneva, 2013.

[2] H. Ginsburg, and E. Deharo, "A call for using natural compounds in the development of new antimalarial treatments-an introduction”, Malaria J, vol.10, 51-58, 2011.

[3] S. B. O. Ovenden, M. Cobbe, R. Kissell, G. W. Birrell, M. Chavchich, and M. D. Edstein, "Phenolic glycosides with antimalarial activity from GreWillea "PoorindaQueen", J Natural Product, vol.74, 74-78, 2011.
[4] R. Batista, A. D. J. Silva, and A. B. Oliveira, "Plant-derived antimalarial agents: new leads and efficient phytomedicines. Part II. Non-alkaloidal natural products", Molecules, vol. 14, 3037-3072, 2009.

[5] K. Na-Bangchang, and J. Karbwang, "Current status of malaria chemotherapy and the role of pharmacology in antimalarial drug research and development", Fundamental Clinical Pharmacol, vol.23, 387-409, 2009.

[6] K. Mishra, A. P. Dash, B. K. Swain and N. Dey, “Antimalarial activities of Andrographis paniculata and Hedyotiscorymbosa extracts and their combination with curcumin", Malaria J, vol.8, 26-37, 2009.

[7] G. Verma, V. K. Dua, D. D. Agarwal, P. and K. Atul, "Antimalarial activity of Holarrhena antidysenterica and Viola canescens, plants traditionally used against malaria in the Garhwal region of north-west Himalaya" Malar. J., vol.10, 20 $25,2011$.

[8] I. Shittu, A. Emmanuel and J. A. Nok, "Antimalaria effect of the ethanolic stem bark extracts of Ficusplatyphylla", Journal Parasitology Research, vol. 2011, 1-5, 2011.

[9] A. Mesfin, M. Giday, A. Animut, and T. Teklehaymanot, "Ethnobotanical study of antimalarial plants in Shinile District, Somali Region, Ethiopia, and in vivo evaluation of selected ones against Plasmodium berghei", JEthnopharmacol, vol. 139, 221-227, 2012.

[10] W. Peters, H. Portus, and L. Robinson "The four day suppressive in vivo antimalarial test" Annals of Tropical Medicine”, Parasitology, vol. 69, 155-171, 1975.

[11] Y. Chen, S. Li, F. Sun, H. Han, X. Zhang, Y. Fan, G. Tai and Y. Zhou, "In vivo antimalarial activities of glycoalkaloids isolated from Solanaceae plants", Pharm Biol, vol. 48, 10181024, 2010.

[12] A. U. Krettli, J. O. Adebayo and L. G. Krettli, "Testing of natural products and synthetic molecules aiming at new antimalarials", Curr Drug Targets, vol.10, 261-270, 2009.

[13] Y. Tadesse, "Bioactivity guided study on the antimalarial activities of Clerodendrum myricoides and Dodonaea angustifolia", Biology Thesis, Addis Ababa University, Ethiopia, 2011.

[14] T. Deressa, Y. Mekonnen and A. Animut, "In vivo antimalarial activities of Clerodendrum myricoides, Dodonaea angustifolia and Aloe debrana against plasmodium berghei", Ethiopian J Health Development, vol. 24, 25-29, 2010.

[15] F. W. Muregi, S. C. Chhabra, E. N. Njagi, C. C. LangatThoruwa, W. M. Njue, A. S. S. Orago, S. A Omar and I. O. Ndiege "Antiplasmodial activity of some Kenyan medicinal plant extracts singly and in combination with chloroquine", Phytotherapy Research, vol. 18, 379-384, 2004. 